Journal Club

Editor's Note: These short, critical reviews of recent papers in the Journal, written exclusively by graduate students or postdoctoral fellows, are intended to summarize the important findings of the paper and provide additional insight and commentary. For more information on the format and purpose of the Journal Club, please see http://www.jneurosci.org/site/misc/ifa_features.xhtml.

\title{
Where Is the Anterior Temporal Lobe and What Does It Do?
}

\author{
Michael F. Bonner ${ }^{1}$ and Amy R. Price ${ }^{1,2}$ \\ ${ }^{1}$ Department of Neurology and ${ }^{2}$ Neuroscience Graduate Group, University of Pennsylvania, Philadelphia, Pennsylvania 19104 \\ Review of Peelen and Caramazza
}

The anterior temporal lobe (ATL) is thought to be critical for semantic memoryour knowledge of objects, people, words, and facts. However, there is substantial disagreement over the precise role of the ATL in semantic memory, and there is considerable variability in the anatomic findings that link the ATL with semantic processing. The inconsistent findings across studies may be related to the diverse anatomic structures within the ATL and their differential contribution to distinct modalities of semantic information (e.g., visual, auditory, affective).

Much of the evidence implicating the ATL in semantic memory has come from neuropsychology. In particular, patients with semantic dementia, a neurodegenerative disease affecting the ATL, exhibit a profound deficit in semantic knowledge with a relative sparing of most other cognitive domains (Warrington, 1975; Patterson et al., 2007). These patients have little trouble performing episodic recall tasks, visual perceptual tasks, or numerical tasks, and can even retain complex behaviors like performing novel musical pieces-yet they have a striking impairment on nearly all assessments of semantic knowledge. Given the broad anatomic extent of disease in semantic dementia,

Received Jan. 4, 2013; revised Jan. 16, 2013; accepted Jan. 21, 2013.

This work was supported by National Institutes of Health Grant AG017586. We thank Murray Grossman and Jonathan Peelle for helpful comments on this manuscript.

Correspondence should be addressed to Michael F. Bonner, Department of Neurology - 2 Gibson, University of Pennsylvania, 3400 Spruce Street, Philadelphia, PA 19104. E-mail: michafra@mail.med.upenn.edu.

DOI:10.1523/JNEUROSCI.0041-13.2013

Copyright $\odot 2013$ the authors $\quad 0270-6474 / 13 / 304213-03 \$ 15.00 / 0$ these findings have implicated a large region of the ATL in semantic memory.

One might expect that complementary functional neuroimaging studies would provide a more fine-grained picture of ATL function. Unfortunately, the evidence from functional neuroimaging has not clearly pointed to the ATL as a critical region for conceptual knowledge. Indeed, fMRI examinations of semantic memory have implicated a broad cortical network in conceptual processing, with sensory and motor regions contributing to representations of the perceptual and action features of concepts (Martin, 2007), and inferior parietal and posterolateral temporal regions supporting multimodal conceptual information (Binder and Desai, 2011). Inconsistent ATL activation can partly be explained by imaging artifacts near the sinuses in fMRI, which may degrade signal detection in the ATL. Nonetheless, the variability of the evidence to date has left many unanswered questions about the functions of ATL structures.

In a recent report in the Journal of Neuroscience, Peelen and Caramazza (2012) provide fMRI evidence that a region of the ventral temporal pole encodes information about the abstract conceptual properties of objects. Their findings complement neuropsychological studies of the ATL and fit within a broad theoretical framework of perception and memory, in which the ventral visual stream processes a hierarchy of increasingly complex information, culminating in anterior regions of the temporal lobe where abstract conceptual properties of objects are represented (Simmons and Barsalou, 2003). This work has implications for our understanding of how ATL structures differentially contribute to semantic memory, and how the semantic system is shaped by the modalities of the information it processes.

In their study, subjects viewed images of objects. Half were objects that are typically found in a kitchen (e.g., pepper mill), and half in a garage (e.g., staple gun). Half were objects that are typically acted upon by rotating (e.g., pepper mill), and half by squeezing (e.g., staple gun). Subjects performed a one-back task, indicating whether they saw two objects in a row that shared a particular conceptual feature (shared location for half of the trials, shared action for the other half). The authors reasoned that if a cortical region encodes abstract conceptual features, it should have a similar neural response to objects with a shared conceptual feature, even if those objects are different in all other respects. They tested this hypothesis using multivoxel pattern analysis to look for regions where objects with a shared conceptual feature had highly similar patterns of fMRI responses. This was performed in regions of interest along the ventral temporal lobe, and in whole-brain searchlight analyses.

These analyses revealed a graded effect along the posterior-anterior axis of the ventral temporal lobe, with the highest degree of similarity in bilateral ventral temporal pole (this anatomic region is illustrated in Fig. 1). A similar effect was observed for both the location and action features of concepts. These effects were 


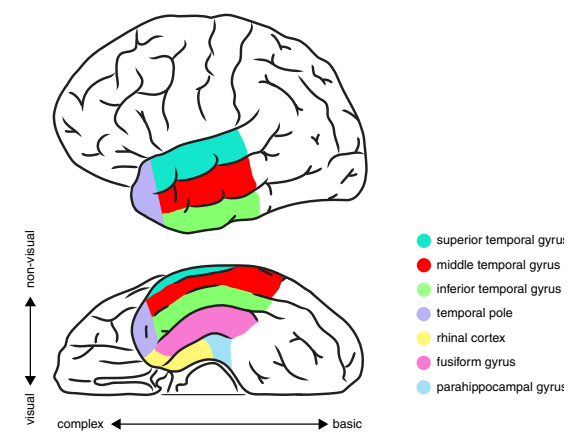

Figure 1. Structures of the anterior temporal lobe. The axes indicate theoretical gradients of differential semantic processes. Along one axis, ventral structures contribute more to the processing of visual information (e.g., object concepts), and dorsolateral structures contribute more to the processing of non-visual information (e.g., abstract concepts, auditory concepts). Along the second axis, posterior structures contribute more to basic object representations, and anterior structures contribute more to the representation of complex conceptual associations.

present regardless of whether the task probed location or action knowledge, suggesting that the responses were not purely task-driven. By comparison, visual similarity across the stimuli was encoded in early visual processing regions of temporal-occipital cortex. Altogether, these findings illustrate a model of hierarchical processing along the ventral visual stream, with lower-level visual-perceptual features processed in temporal-occipital cortex, and higher-level conceptual associations processed in more anterior ventral temporal regions.

Given the anatomic variability of previous ATL findings, it is worthwhile considering the precise anatomy of Peelen and Caramazza's (2012) results in light of specific hypotheses about ATL structures. Until recently, the most prominent accounts of ATL function focused largely on the temporal pole, the most anterior portion of the ATL (Patterson et al., 2007). But more recent work has placed an increasing emphasis on ventral and lateral temporal regions that are posterior to the temporal pole, including the fusiform and parahippocampal gyri (Mion et al., 2010) and the middle and superior temporal gyri (Visser et al., 2010). All of these structures of the ATL are illustrated in Figure 1. Across many studies, these various anatomic structures have been referred to as the "anterior temporal lobe" and ascribed a similar function: serving as an amodal hub that integrates the information associated with a concept (Patterson et al., 2007). The term amodal suggests that the ATL represents all modalities of conceptual information (e.g., object concepts, action concepts, sound concepts) through all modes of reception and expression (e.g., language, photos, sounds). However, the ATL is a large, heterogeneous region containing numerous cytoarchitectonically distinct cortical areas (Brodmann, 1909; Blaizot et al., 2010) with a structured pattern of differential anatomic connectivity (Kondo et al., 2003). It is unlikely that these anatomically distinct regions are functionally equivalent. Furthermore, the spatial resolution of functional neuroimaging techniques is more than adequate for identifying the differential contribution of these regions to semantic processing.

One plausible account for the anatomic variability in previous studies is that there is some degree of modalityspecificity in the functions of the ATL (Yi et al., 2007), and more broadly throughout the temporal lobe (Bonner and Grossman, 2012). In other words, semantic processing in the ATL may not be fully amodal. Indeed, the results of a recent meta-analysis are consistent with this perspective, demonstrating that visual object processing often recruits ventral ATL structures, while linguistic and auditory processing tend to recruit lateral ATL structures (Visser et al., 2010). This metaanalysis focused on the modality of the stimulus materials (e.g., pictures vs words), and did not directly address questions about the modality of the semantic information being processed (e.g., visual concepts, action concepts, sounds concepts). Nonetheless, the findings indicate that there is some degree of modalityspecificity in the semantic functions of the ATL. In this vein, it is relevant that the stimuli used by Peelen and Caramazza (2012) depended on visual perception. This may explain in part why their effects were located in the ventral rather than lateral ATL, as the ventral ATL appears to process predominantly visual information (Visser et al., 2010).

We can also consider Peelen and Caramazza's (2012) findings in relation to the posterior-anterior axis of the ATL. It is worth noting that their strongest effects were in the most anterior region of the ATL-the temporal pole-and not in regions more posterior to this, like the fusiform or parahippocampal gyri (Mion et al., 2010). One relevant hypothesis proposes a posterior-to-anterior gradient in the specificity of semantic processing in the ATL, with more fine-grained semantic information relying more on anterior regions (Tyler et al., 2004). Though Peelen and Caramazza (2012) did not examine multiple levels of semantic specificity in their experiment, their results may nonetheless be consistent with this account. Their task assessed knowledge of how individual objects are used and where they are located. This task may have relied on highly specific semantic information in the most anterior regions of the temporal lobe, whereas a less specific semantic task, like lexical decision, might rely more on the fusiform or parahippocampal gyri.

Finally, it is important to consider the modality of the semantic content that was probed in Peelen and Caramazza's (2012) study. Their experiment examined the conceptual processing of objects, a category of concepts with strongly associated visual features. They reasoned that their ATL findings reflect an extension of the processing hierarchy in the ventral visual stream. However, this interpretation differs from the hypothesis that the ATL is an amodal semantic hub (Patterson et al., 2007). From the amodal perspective, it is proposed that the ATL represents all categories of concepts, including those with weak visual feature associations, such as abstract concepts like "truth" and auditory concepts like "thunder." Yet, critically, most studies of ATL function have focused on the representation of object concepts, which depend heavily on visual feature knowledge. As with Peelen and Caramazza's (2012) study, these investigations do not address the question of whether subregions of the ATL differentially contribute to representations of visual and non-visual concepts. Indeed, the strong focus on object concepts in the literature may have partly biased findings to more ventral temporal regions. It will be important to know in future studies whether there is some degree of modalityspecificity in the semantic processes of the ATL, with ventral temporal regions contributing disproportionately to visual concepts.

A careful consideration of the anatomic subregions of the ATL can inform our understanding of the neural basis of semantic memory. We suggest that Peelen and Caramazza's (2012) findings on object concepts in vision fit within a more fine-grained anatomic hypothesis that proposes modality-specific functions within the substructures of the ATL. In particular, this hypothesis relates visual semantic representations to ventral regions of the temporal pole and the fusiform and parahippocampal gyri. This interpretation contrasts with a purely amodal account of ATL function, and it raises important questions about the functional specificity of ATL substructures. Future studies can address these issues by 
considering where neuroimaging findings fall within the ventral-lateral and posterioranterior axes of the ATL, and how the anatomy of these findings relates to the modality-specific attributes of experimental stimuli.

\section{References}

Binder JR, Desai RH (2011) The neurobiology of semantic memory. Trends in Cognitive Sciences 15:527-536. CrossRef Medline

Blaizot X, Mansilla F, Insausti AM, Constans JM, Salinas-Alamán A, Pró-Sistiaga P, MohedanoMoriano A, Insausti R (2010) The human parahippocampal region: I. Temporal pole cytoarchitectonic and MRI correlation. Cereb Cortex 20:2198-2212. CrossRef Medline

Bonner MF, Grossman M (2012) Gray matter density of auditory association cortex relates to knowledge of sound concepts in primary progressive aphasia. J Neurosci 32:7986-7991. CrossRef Medline

Brodmann K (1909) Vergleichende Lokalisa- tionslehre der Großhirnrinde in ihren Prinzipien dargestellt auf Grund des Zellenbaues. Leipzig: Barth.

Kondo H, Saleem KS, Price JL (2003) Differential connections of the temporal pole with the orbital and medial prefrontal networks in macaque monkeys. J Comp Neurol 465:499-523. CrossRef Medline

Martin A (2007) The representation of object concepts in the brain. Annu Rev Psychol 58: 25-45. CrossRef Medline

Mion M, Patterson K, Acosta-Cabronero J, Pengas G, Izquierdo-Garcia D, Hong YT, Fryer TD, Williams GB, Hodges JR, Nestor PJ (2010) What the left and right anterior fusiform gyri tell us about semantic memory. Brain 133:3256-3268. CrossRef Medline

Patterson K, Nestor PJ, Rogers TT (2007) Where do you know what you know? The representation of semantic knowledge in the human brain. Nat Rev Neurosci 8:976-987. CrossRef Medline

Peelen MV, Caramazza A (2012) Conceptual object representations in human anterior temporal cortex. J Neurosci 32:15728-15736. CrossRef Medline

Simmons WK, Barsalou LW (2003) The similarity-in-topography principle: reconciling theories of conceptual deficits. Cogn Neuropsychol 20:451-486. CrossRef Medline

Tyler LK, Stamatakis EA, Bright P, Acres K, Abdallah S, Rodd JM, Moss HE (2004) Processing objects at different levels of specificity. J Cogn Neurosci 16:351-362. CrossRef Medline

Visser M, Jefferies E, Lambon Ralph MA (2010) Semantic processing in the anterior temporal lobes: a meta-analysis of the functional neuroimaging literature. J Cogn Neurosci 22: 1083-1094. Medline

Warrington EK (1975) The selective impairment of semantic memory. Q J Exp Psychol 27:635-657. CrossRef Medline

Yi HA, Moore P, Grossman M (2007) Reversal of the concreteness effect for verbs in semantic dementia. Neuropsychology 21:9-19. CrossRef Medline 